\title{
A New Fractional Subequation Method and Its Applications for Space-Time Fractional Partial Differential Equations
}

\author{
Fanwei Meng' and Qinghua Feng ${ }^{2}$ \\ ${ }^{1}$ School of Mathematical Sciences, Qufu Normal University, Qufu, Shandong 273165, China \\ ${ }^{2}$ School of Science, Shandong University of Technology, Zibo, Shandong 255049, China \\ Correspondence should be addressed to Qinghua Feng; fqhua@sina.com
}

Received 22 February 2013; Accepted 1 July 2013

Academic Editor: Magdy A. Ezzat

Copyright (c) 2013 F. Meng and Q. Feng. This is an open access article distributed under the Creative Commons Attribution License, which permits unrestricted use, distribution, and reproduction in any medium, provided the original work is properly cited.

\begin{abstract}
A new fractional subequation method is proposed for finding exact solutions for fractional partial differential equations (FPDEs). The fractional derivative is defined in the sense of modified Riemann-Liouville derivative. As applications, abundant exact solutions including solitary wave solutions as well as periodic wave solutions for the space-time fractional generalized Hirota-Satsuma coupled KdV equations are obtained by using this method.
\end{abstract}

\section{Introduction}

Fractional differential equations are generalizations of classical differential equations of integer order. Recently, fractional differential equations have been the focus of many studies due to their frequent appearance in various applications in physics, biology, engineering, signal processing, systems identification, control theory, finance, and fractional dynamics. Among the investigations for fractional differential equations, research for seeking exact solutions and approximate solutions of fractional differential equations is a hot topic. New exact solutions for fractional differential equations may help to understand better corresponding nonlinear wave phenomena they describe. Some powerful methods have been proposed so far (e.g., see [1-12]). Using these methods, a variety of fractional differential equations have been investigated.

In this paper, we propose a new fractional subequation method to establish exact solutions for fractional partial differential equations (FPDEs), which is based on the following fractional ordinary differential equation:

$$
D_{\xi}^{2 \alpha} G(\xi)+\lambda D_{\xi}^{\alpha} G(\xi)+\mu G(\xi)=0, \quad 0<\alpha \leq 1,
$$

where $D_{\xi}^{\alpha} G(\xi)$ denotes the modified Riemann-Liouville derivative of order $\alpha$ for $G(\xi)$ with respect to $\xi$.
The definition and some important properties for Jumarie's modified Riemann-Liouville derivative of order $\alpha$ are listed as follows (see [13-16]):

$$
\begin{aligned}
& D_{t}^{\alpha} f(t) \\
& =\left\{\begin{array}{cc}
\frac{1}{\Gamma(1-\alpha)} \frac{d}{d t} \int_{0}^{t}(t-\xi)^{-\alpha}(f(\xi)-f(0)) d \xi \\
\left(f^{(n)}(t)\right)^{(\alpha-n)}, & 0<\alpha<1,
\end{array}\right\} \begin{array}{c}
n \leq n+1, n \geq 1, \\
D_{t}^{\alpha} t^{r}=\frac{\Gamma(1+r)}{\Gamma(1+r-\alpha)} t^{r-\alpha},
\end{array} \\
& D_{t}^{\alpha}(f(t) g(t))=g(t) D_{t}^{\alpha} f(t)+f(t) D_{t}^{\alpha} g(t),
\end{aligned}
$$

We organize this paper as follows. In Section 2, we derive the expression for $D_{\xi}^{\alpha} G(\xi) / G(\xi)$ related to (1). In Section 3, we give the description of the fractional subequation method for solving FPDEs. Then in Section 4 we apply this method to establish exact solutions for the space-time fractional generalized Hirota-Satsuma coupled KdV equations. Some conclusions are presented at the end of the paper. 


\section{The General Expression for $D_{\xi}^{\alpha} G(\xi) / G(\xi)$}

In order to obtain the general solutions for (1), we suppose $G(\xi)=H(\eta)$ and a nonlinear fractional complex transformation $\eta=\xi^{\alpha} / \Gamma(1+\alpha)$. Then, by (3) and the first equality in (5), (1) can be turned into the following second ordinary differential equation:

$$
H^{\prime \prime}(\eta)+\lambda H^{\prime}(\eta)+\mu H(\eta)=0 .
$$

By the general solutions of (6), we have

$$
\frac{H^{\prime}(\eta)}{H(\eta)}= \begin{cases}-\frac{\lambda}{2}+\frac{\sqrt{\lambda^{2}-4 \mu}}{2}\left(\frac{C_{1} \sinh \left(\sqrt{\lambda^{2}-4 \mu} / 2\right) \eta+C_{2} \cosh \left(\sqrt{\lambda^{2}-4 \mu} / 2\right) \eta}{C_{1} \cosh \left(\sqrt{\lambda^{2}-4 \mu} / 2\right) \eta+C_{2} \sinh \left(\sqrt{\lambda^{2}-4 \mu} / 2\right) \eta}\right), & \lambda^{2}-4 \mu>0, \\ -\frac{\lambda}{2}+\frac{\sqrt{4 \mu-\lambda^{2}}}{2}\left(\frac{-C_{1} \sin \left(\sqrt{4 \mu-\lambda^{2}} / 2\right) \eta+C_{2} \cos \left(\sqrt{4 \mu-\lambda^{2}} / 2\right) \eta}{C_{1} \cos \left(\sqrt{4 \mu-\lambda^{2}} / 2\right) \eta+C_{2} \sin \left(\sqrt{4 \mu-\lambda^{2}} / 2\right) \eta}\right), & \lambda^{2}-4 \mu<0, \\ -\frac{\lambda}{2}+\frac{C_{2}}{C_{1}+C_{2} \eta}, & \lambda^{2}-4 \mu=0,\end{cases}
$$

where $C_{1}$ and $C_{2}$ are arbitrary constants. obtain

$$
\frac{D_{\xi}^{\alpha} G(\xi)}{G(\xi)}= \begin{cases}-\frac{\lambda}{2}+\frac{\sqrt{\lambda^{2}-4 \mu}}{2}\left(\frac{C_{1} \sinh \left(\sqrt{\lambda^{2}-4 \mu} / 2 \Gamma(1+\alpha)\right) \xi^{\alpha}+C_{2} \cosh \left(\sqrt{\lambda^{2}-4 \mu} / 2 \Gamma(1+\alpha)\right) \xi^{\alpha}}{C_{1} \cosh \left(\sqrt{\lambda^{2}-4 \mu} / 2 \Gamma(1+\alpha)\right) \xi^{\alpha}+C_{2} \sinh \left(\sqrt{\lambda^{2}-4 \mu} / 2 \Gamma(1+\alpha)\right) \xi^{\alpha}}\right), & \lambda^{2}-4 \mu>0, \\ -\frac{\lambda}{2}+\frac{\sqrt{4 \mu-\lambda^{2}}}{2}\left(\frac{-C_{1} \sin \left(\sqrt{4 \mu-\lambda^{2}} / 2 \Gamma(1+\alpha)\right) \xi^{\alpha}+C_{2} \cos \left(\sqrt{4 \mu-\lambda^{2}} / 2 \Gamma(1+\alpha)\right) \xi^{\alpha}}{C_{1} \cos \left(\sqrt{4 \mu-\lambda^{2}} / 2 \Gamma(1+\alpha)\right) \xi^{\alpha}+C_{2} \sin \left(\sqrt{4 \mu-\lambda^{2}} / 2 \Gamma(1+\alpha)\right) \xi^{\alpha}}\right), & \lambda^{2}-4 \mu<0, \\ -\frac{\lambda}{2}+\frac{C_{2} \Gamma(1+\alpha)}{C_{1} \Gamma(1+\alpha)+C_{2} \xi^{\alpha}}, & \lambda^{2}-4 \mu=0 .\end{cases}
$$

\section{Description of the Fractional Subequation Method}

In this section, we give the main steps of the fractional subequation method for finding exact solutions for FPDEs.

Suppose that an FPDE, say in the independent variables $t, x_{1}, x_{2}, \ldots, x_{n}$, is given by

$$
\begin{gathered}
P\left(u_{1}, \ldots, u_{k}, D_{t}^{\alpha} u_{1}, \ldots, D_{t}^{\alpha} u_{k}, D_{x_{1}}^{\alpha} u_{1}, \ldots,\right. \\
D_{x_{1}}^{\alpha} u_{k}, \ldots, D_{x_{n}}^{\alpha} u_{1}, \ldots, D_{x_{n}}^{\alpha} u_{k}, D_{t}^{2 \alpha} u_{1}, \ldots, \\
\left.D_{t}^{2 \alpha} u_{k}, D_{x_{1}}^{2 \alpha} u_{1}, \ldots\right)=0
\end{gathered}
$$

where $u_{i}=u_{i}\left(t, x_{1}, x_{2}, \ldots, x_{n}\right), i=1, \ldots, k$, are unknown functions and $P$ is a polynomial in $u_{i}$ and their various partial derivatives including fractional derivatives.
Step 1. Suppose that

$$
\begin{gathered}
u_{i}\left(t, x_{1}, x_{2}, \ldots, x_{n}\right)=U_{i}(\xi), \\
\xi=c t+k_{1} x_{1}+k_{2} x_{2}+\cdots+k_{n} x_{n}+\xi_{0} .
\end{gathered}
$$

Then, by the second equality in (5), (9) can be turned into the following fractional ordinary differential equation with respect to the variable $\xi$ :

$$
\begin{aligned}
\widetilde{P} & \left(U_{1}, \ldots, U_{k}, c^{\alpha} D_{\xi}^{\alpha} U_{1}, \ldots, c^{\alpha} D_{\xi}^{\alpha} U_{k},\right. \\
& k_{1}^{\alpha} D_{\xi}^{\alpha} U_{1}, \ldots, k_{1}^{\alpha} D_{\xi}^{\alpha} U_{k}, \ldots, k_{n}^{\alpha} D_{\xi}^{\alpha} U_{1}, \ldots, \\
& k_{n}^{\alpha} D_{\xi}^{\alpha} U_{k}, c^{2 \alpha} D_{\xi}^{2 \alpha} U_{1}, \ldots, \\
& \left.c^{2 \alpha} D_{\xi}^{2 \alpha} U_{k}, k_{1}^{2 \alpha} D_{\xi}^{2 \alpha} U_{1}, \ldots\right)=0 .
\end{aligned}
$$


Step 2. Suppose that the solution of (11) can be expressed by a polynomial in $\left(D_{\xi}^{\alpha} G / G\right)$ as follows:

$$
\begin{array}{r}
U_{j}(\xi) \\
=a_{j, 0}+\sum_{i=1}^{m_{j}}\left[a_{j, i}\left(\frac{D_{\xi}^{\alpha} G}{G}\right)^{i}\right. \\
\left.+b_{j, i}\left(\frac{D_{\xi}^{\alpha} G}{G}\right)^{i-1} \sqrt{\sigma\left(1+\frac{1}{\mu}\left(\frac{D_{\xi}^{\alpha} G}{G}\right)^{2}\right)}\right], \\
j=1,2, \ldots, k,
\end{array}
$$

where $G=G(\xi)$ satisfies (1), $\sigma$ is a constant, and $a_{j, i}, i=$ $0,1, \ldots, m, j=1,2, \ldots, k$, are constants to be determined later. The positive integer $m$ can be determined by considering the homogeneous balance between the highest-order derivatives and nonlinear terms appearing in (11).

Step 3. Substituting (12) into (11), using (1), and collecting all terms with the same order of $\left(D_{\xi}^{\alpha} G /\right.$ $G) \sqrt{\sigma\left(1+(1 / \mu)\left(D_{\xi}^{\alpha} G / G\right)^{2}\right)}$ together, the left-hand side of (11) is converted into another polynomial in $\left(D_{\xi}^{\alpha} G / G\right)$. Equating each coefficient of this polynomial to zero yields a set of algebraic equations for $a_{j 0}, a_{j, i}, b_{j, i}, i=1, \ldots, m$, $j=1,2, \ldots, k$.

Step 4. Solving the equations in Step 3 and using (8), we can construct a variety of exact solutions for (9).

\section{Application of \\ the Method to Space-Time Fractional Generalized Hirota-Satsuma Coupled KdV Equations}

In this section, we will apply the described method in Section 3 to solve the space-time fractional generalized Hirota-Satsuma coupled KdV equations $[15,16]$ :

$$
\begin{gathered}
D_{t}^{\alpha} u-\frac{1}{2} D_{x}^{3 \alpha} u+3 u D_{x}^{\alpha} u-3 D_{x}^{\alpha}(v w)=0, \\
D_{t}^{\alpha} v+D_{x}^{3 \alpha} v-3 u D_{x}^{\alpha} v=0 \\
D_{t}^{\alpha} w+D_{x}^{3 \alpha} w-3 u D_{x}^{\alpha} w=0, \\
0<\alpha \leq 1 .
\end{gathered}
$$

Equations (13) can be used to describe the interaction of two long waves with different dispersion relations [17]. In [15], the authors solved equations (13) by a proposed fractional subequation method based on the fractional Riccati equation, while in [16], (13) are solved by the known $\left(G^{\prime} / G\right)$-expansion method. Now we apply the described method in Section 3 to solve (13). To begin with, suppose that $u(x, t)=U(\xi), v(x, t)=$ $V(\xi), w(x, t)=W(\xi)$, where $\xi=k x+c t+\xi_{0}, k, c, \xi_{0}$ are all constants with $k, c \neq 0$. Then, by usinge the second equality in (4), we obtain

$$
\begin{aligned}
& D_{x}^{\alpha} u=D_{x}^{\alpha} U(\xi)=\left(D_{\xi}^{\alpha} U\right)\left(\xi_{x}^{\prime}\right)^{\alpha}=k^{\alpha} D_{\xi}^{\alpha} U, \\
& D_{t}^{\alpha} u=D_{t}^{\alpha} U(\xi)=\left(D_{\xi}^{\alpha} U\right)\left(\xi_{t}^{\prime}\right)^{\alpha}=c^{\alpha} D_{\xi}^{\alpha} U,
\end{aligned}
$$

and similarly we have

$$
\begin{aligned}
D_{x}^{\alpha} v=k^{\alpha} D_{\xi}^{\alpha} V, & D_{t}^{\alpha} v=c^{\alpha} D_{\xi}^{\alpha} V, \\
D_{x}^{\alpha} w=k^{\alpha} D_{\xi}^{\alpha} W, & D_{t}^{\alpha} w=c^{\alpha} D_{\xi}^{\alpha} W
\end{aligned}
$$

then (11) can be turned into the following fractional ordinary differential equations with respect to the variable $\xi$ :

$$
\begin{aligned}
c^{\alpha} D_{\xi}^{\alpha} U- & \frac{1}{2} k^{3 \alpha} D_{\xi}^{3 \alpha} U+3 k^{\alpha} U D_{\xi}^{\alpha} U-3 k^{\alpha} D_{\xi}^{\alpha}(V W)=0, \\
& c^{\alpha} D_{\xi}^{\alpha} V+k^{3 \alpha} D_{\xi}^{3 \alpha} V-3 k^{\alpha} U D_{\xi}^{\alpha} V=0, \\
& c^{\alpha} D_{\xi}^{\alpha} W+k^{3 \alpha} D_{\xi}^{3 \alpha} W-3 k^{\alpha} U D_{\xi}^{\alpha} W=0 .
\end{aligned}
$$

Suppose that the solutions of (16) can be expressed by

$$
\begin{aligned}
& U(\xi)=a_{0}+\sum_{i=1}^{m_{1}}\left[a_{i}\left(\frac{D_{\xi}^{\alpha} G}{G}\right)^{i}\right. \\
& \left.+b_{i}\left(\frac{D_{\xi}^{\alpha} G}{G}\right)^{i-1} \sqrt{\sigma\left(1+\frac{1}{\mu}\left(\frac{D_{\xi}^{\alpha} G}{G}\right)^{2}\right)}\right] \text {, } \\
& V(\xi)=c_{0}+\sum_{i=1}^{m_{2}}\left[c_{i}\left(\frac{D_{\xi}^{\alpha} G}{G}\right)^{i}\right. \\
& \left.+d_{i}\left(\frac{D_{\xi}^{\alpha} G}{G}\right)^{i-1} \sqrt{\sigma\left(1+\frac{1}{\mu}\left(\frac{D_{\xi}^{\alpha} G}{G}\right)^{2}\right)}\right], \\
& W(\xi)=e_{0}+\sum_{i=1}^{m_{3}}\left[e_{i}\left(\frac{D_{\xi}^{\alpha} G}{G}\right)^{i}\right. \\
& \left.+f_{i}\left(\frac{D_{\xi}^{\alpha} G}{G}\right)^{i-1} \sqrt{\sigma\left(1+\frac{1}{\mu}\left(\frac{D_{\xi}^{\alpha} G}{G}\right)^{2}\right)}\right] .
\end{aligned}
$$

Balancing the order of $D_{\xi}^{3 \alpha} U$ and $D_{\xi}^{\alpha}(V W), D_{\xi}^{3 \alpha} V$ and $U D_{\xi}^{\alpha} V$, and $D_{\xi}^{3 \alpha} W$ and $U D_{\xi}^{\alpha} W$ in (16), we have $m_{1}=m_{2}=m_{3}=2$. So

$$
\begin{aligned}
U(\xi)= & a_{0}+a_{1}\left(\frac{D_{\xi}^{\alpha} G}{G}\right)+a_{2}\left(\frac{D_{\xi}^{\alpha} G}{G}\right)^{2} \\
& +b_{1} \sqrt{\sigma\left(1+\frac{1}{\mu}\left(\frac{D_{\xi}^{\alpha} G}{G}\right)^{2}\right)} \\
& +b_{2}\left(\frac{D_{\xi}^{\alpha} G}{G}\right) \sqrt{\sigma\left(1+\frac{1}{\mu}\left(\frac{D_{\xi}^{\alpha} G}{G}\right)^{2}\right)},
\end{aligned}
$$




$$
\begin{aligned}
V(\xi)= & c_{0}+c_{1}\left(\frac{D_{\xi}^{\alpha} G}{G}\right)+c_{2}\left(\frac{D_{\xi}^{\alpha} G}{G}\right)^{2} \\
& +d_{1} \sqrt{\sigma\left(1+\frac{1}{\mu}\left(\frac{D_{\xi}^{\alpha} G}{G}\right)^{2}\right)} \\
& +d_{2}\left(\frac{D_{\xi}^{\alpha} G}{G}\right) \sqrt{\sigma\left(1+\frac{1}{\mu}\left(\frac{D_{\xi}^{\alpha} G}{G}\right)^{2}\right)}, \\
W(\xi)= & e_{0}+e_{1}\left(\frac{D_{\xi}^{\alpha} G}{G}\right)+e_{2}\left(\frac{D_{\xi}^{\alpha} G}{G}\right)^{2} \\
& +f_{1} \sqrt{\sigma\left(1+\frac{1}{\mu}\left(\frac{D_{\xi}^{\alpha} G}{G}\right)^{2}\right)} \\
& +f_{2}\left(\frac{D_{\xi}^{\alpha} G}{G}\right) \sqrt{\sigma\left(1+\frac{1}{\mu}\left(\frac{D_{\xi}^{\alpha} G}{G}\right)^{2}\right)} .
\end{aligned}
$$

Substituting (18) into (16), using (1), and collecting all the terms with the same power of $\left(D_{\xi}^{\alpha} G / G\right)$ $\sqrt{\sigma\left(1+(1 / \mu)\left(D_{\xi}^{\alpha} G / G\right)^{2}\right)}$ together, equating each coefficient to zero yields a set of algebraic equations. Solving these equations, with the aid of the mathematical software Maple, yields the following seven groups of values.

Case 1. One has

$$
\begin{gathered}
\mu=\frac{1}{4} k^{-4 \alpha} \sigma b_{2}^{2}, \quad \lambda=0, \quad a_{0}=\frac{1}{3} k^{-\alpha} c^{\alpha}+\frac{5}{12} k^{-2 \alpha} \sigma b_{2}^{2}, \\
a_{1}=0, \quad a_{2}=2 k^{2 \alpha}, \quad b_{1}=0, \quad b_{2}=b_{2}, \\
c_{0}=\frac{1}{24} \frac{b_{2}\left(16 k^{-\alpha} c^{\alpha} f_{2}+5 k^{-2 \alpha} f_{2} \sigma b_{2}^{2}-6 e_{0} b_{2}\right)}{f_{2}^{2}}, \quad c_{1}=0, \\
c_{2}=\frac{k^{2 \alpha} b_{2}}{2 f_{2}}, \quad d_{1}=0, \quad d_{2}=\frac{b_{2}^{2}}{4 f_{2}}, \quad e_{0}=e_{0}, \\
e_{1}=0, \quad e_{2}=\frac{2 k^{2 \alpha} f_{2}}{b_{2}}, \quad f_{1}=0, \quad f_{2}=f_{2} .
\end{gathered}
$$

Case 2. One has

$$
\begin{gathered}
\mu=\mu, \quad \lambda=\frac{1}{4} k^{-2 \alpha} a_{1}, \\
a_{0}=\frac{1}{48} k^{-2 \alpha} a_{1}^{2}+\frac{8}{3} k^{2 \alpha} \mu+\frac{1}{3} k^{-\alpha} c^{\alpha}, \\
a_{1}=a_{1}, \quad a_{2}=4 k^{2 \alpha}, \quad b_{1}=0, \quad b_{2}=0,
\end{gathered}
$$

$$
\begin{gathered}
c_{0}=\frac{1}{96} c_{2}\left(k^{-4 \alpha} a_{1}^{2}+128 \mu+64 c^{\alpha} k^{-3 \alpha}-24 k^{-4 \alpha} c_{2} e_{0}\right), \\
c_{1}=\frac{1}{4} c_{2} k^{-2 \alpha} a_{1}, \quad c_{2}=c_{2}, \quad d_{1}=0, \\
d_{2}=0, \quad e_{0}=e_{0}, \quad e_{1}=\frac{k^{2 \alpha} a_{1}}{c_{2}}, \\
e_{2}=\frac{4 k^{4 \alpha}}{c_{2}}, \quad f_{1}=0, \quad f_{2}=0 .
\end{gathered}
$$

Case 3. One has

$$
\begin{gathered}
\mu=\frac{1}{16} k^{-4 \alpha} a_{1}^{2}+c^{\alpha} k^{-3 \alpha}-\frac{3}{4} k^{-4 \alpha} c_{1} e_{1}, \\
\lambda=\frac{1}{2} k^{-2 \alpha} a_{1}, \\
a_{0}=\frac{1}{8} k^{-2 \alpha} a_{1}^{2}+k^{-\alpha} c^{\alpha}-\frac{1}{2} k^{-2 \alpha} c_{1} e_{1}, \\
a_{1}=a_{1}, \quad a_{2}=2 k^{2 \alpha}, \quad b_{1}=0, \\
b_{2}=0, \quad c_{0}=c_{0}, \quad c_{1}=c_{1}, \\
c_{2}=0, \quad d_{1}=0, \quad d_{2}=0, \\
e_{0}=\frac{e_{1}\left(k^{-2 \alpha} a_{1} c_{1}-2 c_{0}\right)}{2 c_{1}}, \quad e_{1}=e_{1}, \\
e_{2}=0, \quad f_{1}=0, \quad f_{2}=0 .
\end{gathered}
$$

Case 4. One has

$$
\begin{gathered}
\mu=k^{-4 \alpha} \sigma b_{2}^{2}, \quad \lambda=0, \\
a_{0}=\frac{2}{3} k^{-2 \alpha} \sigma b_{2}^{2}+\frac{1}{3} k^{-\alpha} c^{\alpha}, \quad a_{1}=0, \\
a_{2}=k^{2 \alpha}, \quad b_{1}=0, \quad b_{2}=b_{2}, \\
c_{0}=c_{0}, \quad c_{1}=\frac{k^{2 \alpha} d_{1}}{b_{2}}, \quad c_{2}=0, \\
e_{0}=\frac{c_{0} b_{2}^{2}\left(k^{-4 \alpha} \sigma b_{2}^{2}-4 c^{\alpha} k^{-3 \alpha}\right)}{12 d_{1}^{2}}, \\
e_{1}=\frac{b_{2}\left(-k^{-2 \alpha} \sigma b_{2}^{2}+4 k^{-\alpha} c^{\alpha}\right)}{12 d_{1}}, \quad e_{2}=0, \\
f_{1}=-\frac{b_{2}^{2}\left(k^{-4 \alpha} \sigma b_{2}^{2}-4 c^{\alpha} k^{-3 \alpha}\right)}{12 d_{1}}, \quad f_{2}=0 .
\end{gathered}
$$

Case 5. One has

$$
\begin{array}{ccc}
\mu=4 c^{\alpha} k^{-3 \alpha}, & \lambda=0, & a_{0}=3 k^{-\alpha} c^{\alpha}, \\
a_{1}=0, & a_{2}=k^{2 \alpha}, & b_{1}=0,
\end{array}
$$




$$
\begin{gathered}
b_{2}= \pm k^{-\alpha} \sqrt{\frac{c^{\alpha} k^{3 \alpha}}{\sigma}}, \quad c_{0}=0, \quad c_{1}=0, \\
c_{2}=0, \quad d_{1}=0, \quad d_{2}=0, \quad e_{0}=e_{0}, \\
e_{1}= \pm \frac{k^{3 \alpha} f_{1}}{2} \sqrt{\frac{\sigma}{c^{\alpha} k^{3 \alpha}}}, \quad e_{2}=0, \\
f_{1}=f_{1}, \quad f_{2}=0 .
\end{gathered}
$$

Case 6. One has

$$
\begin{array}{r}
\mu=\mu, \quad \lambda=0, \quad a_{0}=\frac{2}{3} k^{2 \alpha} \mu+\frac{1}{3} k^{-\alpha} c^{\alpha}, \\
a_{1}=0, \quad a_{2}=2 k^{2 \alpha}, \quad b_{1}=0, \\
b_{2}=0, \quad c_{0}=c_{0}, \quad c_{1}=c_{1}, \\
c_{2}=0, \quad d_{1}=0, \quad d_{2}=0, \\
e_{0}=-\frac{4 c_{0}\left(-k^{4 \alpha} \mu+k^{\alpha} c^{\alpha}\right)}{3 c_{1}^{2}}, \\
e_{1}=\frac{4\left(-k^{4 \alpha} \mu+k^{\alpha} c^{\alpha}\right)}{3 c_{1}}, \\
e_{2}=0, \quad f_{1}=0, \quad f_{2}=0 .
\end{array}
$$

Case 7. One has

$$
\begin{gathered}
\mu=\mu, \quad \lambda=0, \quad a_{0}=\frac{5}{3} k^{2 \alpha} \mu+\frac{1}{3} k^{-\alpha} c^{\alpha}, \\
a_{1}=0, \quad a_{2}=2 k^{2 \alpha}, \quad b_{1}=0, \\
b_{2}=0, \quad c_{0}=c_{0}, \quad c_{1}=0, \\
c_{2}=0, \quad d_{1}=d_{1}, \quad d_{2}=0, \\
e_{0}=-\frac{2 c_{0} \mu\left(k^{4 \alpha} \mu+2 k^{\alpha} c^{\alpha}\right)}{3 \sigma d_{1}^{2}}, \\
e_{1}=0, \quad e_{2}=0, \\
f_{1}=\frac{2 \mu\left(k^{4 \alpha} \mu+2 k^{\alpha} c^{\alpha}\right)}{3\left(\sigma d_{1}\right)}, \quad f_{2}=0 .
\end{gathered}
$$

Substituting the previous results into (18) and combining with (8), we can obtain a series of exact solutions for (13).

From Case 1, we obtain the following exact solutions. When $\mu<0$,

$$
\begin{aligned}
U_{1}(\xi)= & \frac{1}{3} k^{-\alpha} c^{\alpha}+\frac{5}{12} k^{-2 \alpha} \sigma b_{2}^{2}-2 k^{2 \alpha} \mu \\
& \times\left[\left(C_{1} \sinh \left(\frac{\sqrt{-\mu} \xi^{\alpha}}{\Gamma(1+\alpha)}\right)\right.\right.
\end{aligned}
$$

$$
\begin{aligned}
& \left.+C_{2} \cosh \left(\frac{\sqrt{-\mu} \xi^{\alpha}}{\Gamma(1+\alpha)}\right)\right) \\
& \times\left(C_{1} \cosh \left(\frac{\sqrt{-\mu} \xi^{\alpha}}{\Gamma(1+\alpha)}\right)\right. \\
& \left.\left.+C_{2} \sinh \left(\frac{\sqrt{-\mu} \xi^{\alpha}}{\Gamma(1+\alpha)}\right)\right)^{-1}\right]^{2} \\
& +b_{2} \sqrt{-\mu}\left[\left(C_{1} \sinh \left(\frac{\sqrt{-\mu} \xi^{\alpha}}{\Gamma(1+\alpha)}\right)\right.\right.
\end{aligned}
$$

$$
\begin{aligned}
& \left.+C_{2} \cosh \left(\frac{\sqrt{-\mu} \xi^{\alpha}}{\Gamma(1+\alpha)}\right)\right) \\
& \times\left(C_{1} \cosh \left(\frac{\sqrt{-\mu} \xi^{\alpha}}{\Gamma(1+\alpha)}\right)\right. \\
& \left.\left.+C_{2} \sinh \left(\frac{\sqrt{-\mu} \xi^{\alpha}}{\Gamma(1+\alpha)}\right)\right)^{-1}\right]
\end{aligned}
$$$$
\times\left(\sigma \left\{1-\left[\left(C_{1} \sinh \left(\frac{\sqrt{-\mu} \xi^{\alpha}}{\Gamma(1+\alpha)}\right)\right.\right.\right.\right.
$$$$
\left.+C_{2} \cosh \left(\frac{\sqrt{-\mu} \xi^{\alpha}}{\Gamma(1+\alpha)}\right)\right)
$$$$
\times\left(C_{1} \cosh \left(\frac{\sqrt{-\mu} \xi^{\alpha}}{\Gamma(1+\alpha)}\right)\right.
$$

$$
\left.\left.\left.\left.+C_{2} \sinh \left(\frac{\sqrt{-\mu} \xi^{\alpha}}{\Gamma(1+\alpha)}\right)\right)^{-1}\right]^{2}\right\}\right)^{1 / 2}
$$

$$
\begin{array}{r}
V_{1}(\xi)=\frac{1}{24} \frac{b_{2}\left(16 k^{-\alpha} c^{\alpha} f_{2}+5 k^{-2 \alpha} f_{2} \sigma b_{2}^{2}-6 e_{0} b_{2}\right)}{f_{2}^{2}} \\
-\frac{k^{2 \alpha} b_{2}}{2 f_{2}} \mu\left[\left(C_{1} \sinh \left(\frac{\sqrt{-\mu} \xi^{\alpha}}{\Gamma(1+\alpha)}\right)\right.\right. \\
\left.+C_{2} \cosh \left(\frac{\sqrt{-\mu} \xi^{\alpha}}{\Gamma(1+\alpha)}\right)\right) \\
\times\left(C_{1} \cosh \left(\frac{\sqrt{-\mu} \xi^{\alpha}}{\Gamma(1+\alpha)}\right)\right. \\
\left.\left.+C_{2} \sinh \left(\frac{\sqrt{-\mu} \xi^{\alpha}}{\Gamma(1+\alpha)}\right)\right)^{-1}\right]^{2} \\
+\frac{b_{2}^{2}}{4 f_{2}} \sqrt{-\mu}\left[\left(C_{1} \sinh \left(\frac{\sqrt{-\mu} \xi^{\alpha}}{\Gamma(1+\alpha)}\right)\right.\right. \\
\left.\quad+C_{2} \cosh \left(\frac{\sqrt{-\mu} \xi^{\alpha}}{\Gamma(1+\alpha)}\right)\right) \\
\times\left(C_{1} \cosh \left(\frac{\sqrt{-\mu} \xi^{\alpha}}{\Gamma(1+\alpha)}\right)\right.
\end{array}
$$




$$
\begin{aligned}
& \left.\left.+C_{2} \sinh \left(\frac{\sqrt{-\mu} \xi^{\alpha}}{\Gamma(1+\alpha)}\right)\right)^{-1}\right] \\
& \times\left(\sigma \left\{1-\left[\left(C_{1} \sinh \left(\frac{\sqrt{-\mu} \xi^{\alpha}}{\Gamma(1+\alpha)}\right)\right.\right.\right.\right. \\
& \left.+C_{2} \cosh \left(\frac{\sqrt{-\mu} \xi^{\alpha}}{\Gamma(1+\alpha)}\right)\right) \\
& \times\left(C_{1} \cosh \left(\frac{\sqrt{-\mu} \xi^{\alpha}}{\Gamma(1+\alpha)}\right)\right. \\
& \left.\left.\left.+C_{2} \sinh \left(\frac{\sqrt{-\mu} \xi^{\alpha}}{\Gamma(1+\alpha)}\right)\right)^{-1}\right]^{2}\right)^{1 / 2}
\end{aligned}
$$

$$
\begin{array}{r}
W_{1}(\xi)=e_{0}-\frac{2 k^{2 \alpha} f_{2}}{b_{2}} \mu[ \\
+C_{1} \sinh \left(\frac{\sqrt{-\mu} \xi^{\alpha}}{\Gamma(1+\alpha)}\right) \\
\left.+C_{2} \cosh \left(\frac{\sqrt{-\mu} \xi^{\alpha}}{\Gamma(1+\alpha)}\right)\right) \\
\times\left(C_{1} \cosh \left(\frac{\sqrt{-\mu} \xi^{\alpha}}{\Gamma(1+\alpha)}\right)\right. \\
\left.\left.+C_{2} \sinh \left(\frac{\sqrt{-\mu} \xi^{\alpha}}{\Gamma(1+\alpha)}\right)\right)^{-1}\right]^{2}
\end{array}
$$$$
+f_{2} \sqrt{-\mu}\left[\left(C_{1} \sinh \left(\frac{\sqrt{-\mu} \xi^{\alpha}}{\Gamma(1+\alpha)}\right)\right.\right.
$$$$
\left.+C_{2} \cosh \left(\frac{\sqrt{-\mu} \xi^{\alpha}}{\Gamma(1+\alpha)}\right)\right)
$$$$
\times\left(C_{1} \cosh \left(\frac{\sqrt{-\mu} \xi^{\alpha}}{\Gamma(1+\alpha)}\right)\right.
$$$$
\left.\left.+C_{2} \sinh \left(\frac{\sqrt{-\mu} \xi^{\alpha}}{\Gamma(1+\alpha)}\right)\right)^{-1}\right]
$$$$
\times\left(\sigma \left\{1-\left[\left(C_{1} \sinh \left(\frac{\sqrt{-\mu} \xi^{\alpha}}{\Gamma(1+\alpha)}\right)\right.\right.\right.\right.
$$$$
\left.+C_{2} \cosh \left(\frac{\sqrt{-\mu} \xi^{\alpha}}{\Gamma(1+\alpha)}\right)\right)
$$$$
\times\left(C_{1} \cosh \left(\frac{\sqrt{-\mu} \xi^{\alpha}}{\Gamma(1+\alpha)}\right)\right.
$$$$
\left.\left.\left.\left.+C_{2} \sinh \left(\frac{\sqrt{-\mu} \xi^{\alpha}}{\Gamma(1+\alpha)}\right)\right)^{-1}\right]^{2}\right\}\right)^{1 / 2}
$$

where $\xi=k x+c t+\xi_{0}, \mu=(1 / 4) k^{-4 \alpha} \sigma b_{2}^{2}$.
When $\mu>0$,

$$
\begin{aligned}
& U_{2}(\xi)=\frac{1}{3} k^{-\alpha} c^{\alpha}+\frac{5}{12} k^{-2 \alpha} \sigma b_{2}^{2}+2 k^{2 \alpha} \mu \\
& \times\left[\left(-C_{1} \sin \left(\frac{\sqrt{\mu}}{\Gamma(1+\alpha)}\right) \xi^{\alpha}\right.\right. \\
& \left.+C_{2} \cos \left(\frac{\sqrt{\mu}}{\Gamma(1+\alpha)}\right) \xi^{\alpha}\right) \\
& \times\left(C_{1} \cos \left(\frac{\sqrt{\mu}}{\Gamma(1+\alpha)}\right) \xi^{\alpha}\right. \\
& \left.\left.+C_{2} \sin \left(\frac{\sqrt{\mu}}{\Gamma(1+\alpha)}\right) \xi^{\alpha}\right)^{-1}\right]^{2} \\
& +b_{2} \sqrt{\mu}\left[\left(-C_{1} \sin \left(\frac{\sqrt{\mu}}{\Gamma(1+\alpha)}\right) \xi^{\alpha}\right.\right. \\
& \left.+C_{2} \cos \left(\frac{\sqrt{\mu}}{\Gamma(1+\alpha)}\right) \xi^{\alpha}\right) \\
& \times\left(C_{1} \cos \left(\frac{\sqrt{\mu}}{\Gamma(1+\alpha)}\right) \xi^{\alpha}\right. \\
& \left.\left.+C_{2} \sin \left(\frac{\sqrt{\mu}}{\Gamma(1+\alpha)}\right) \xi^{\alpha}\right)^{-1}\right] \\
& \times\left(\sigma \left\{1+\left[\left(-C_{1} \sin \left(\frac{\sqrt{\mu}}{\Gamma(1+\alpha)}\right) \xi^{\alpha}\right.\right.\right.\right. \\
& \left.+C_{2} \cos \left(\frac{\sqrt{\mu}}{\Gamma(1+\alpha)}\right) \xi^{\alpha}\right) \\
& \times\left(C_{1} \cos \left(\frac{\sqrt{\mu}}{\Gamma(1+\alpha)}\right) \xi^{\alpha}\right. \\
& \left.\left.\left.+C_{2} \sin \left(\frac{\sqrt{\mu}}{\Gamma(1+\alpha)}\right) \xi^{\alpha}\right)^{-1}\right]^{2}\right)^{1 / 2}
\end{aligned}
$$

$$
\begin{array}{r}
V_{2}(\xi)=\frac{1}{24} \frac{b_{2}\left(16 k^{-\alpha} c^{\alpha} f_{2}+5 k^{-2 \alpha} f_{2} \sigma b_{2}^{2}-6 e_{0} b_{2}\right)}{f_{2}^{2}} \\
+\frac{k^{2 \alpha} b_{2}}{2 f_{2} \mu}\left[\left(-C_{1} \sin \left(\frac{\sqrt{\mu}}{\Gamma(1+\alpha)}\right) \xi^{\alpha}\right.\right. \\
\left.+C_{2} \cos \left(\frac{\sqrt{\mu}}{\Gamma(1+\alpha)}\right) \xi^{\alpha}\right) \\
\times\left(C_{1} \cos \left(\frac{\sqrt{\mu}}{\Gamma(1+\alpha)}\right) \xi^{\alpha}\right. \\
\left.\left.+C_{2} \sin \left(\frac{\sqrt{\mu}}{\Gamma(1+\alpha)}\right) \xi^{\alpha}\right)^{-1}\right]^{2}
\end{array}
$$$$
+\frac{b_{2}^{2}}{4 f_{2}} \sqrt{\mu}\left[\left(-C_{1} \sin \left(\frac{\sqrt{\mu}}{\Gamma(1+\alpha)}\right) \xi^{\alpha}\right.\right.
$$ 
Journal of Applied Mathematics

7

$$
\begin{gathered}
\left.+C_{2} \cos \left(\frac{\sqrt{\mu}}{\Gamma(1+\alpha)}\right) \xi^{\alpha}\right) \\
\times\left(C_{1} \cos \left(\frac{\sqrt{\mu}}{\Gamma(1+\alpha)}\right) \xi^{\alpha}\right. \\
\left.\left.+C_{2} \sin \left(\frac{\sqrt{\mu}}{\Gamma(1+\alpha)}\right) \xi^{\alpha}\right)^{-1}\right] \\
\times\left(\sigma \left\{1+\left[\left(-C_{1} \sin \left(\frac{\sqrt{\mu}}{\Gamma(1+\alpha)}\right) \xi^{\alpha}\right.\right.\right.\right. \\
\left.+C_{2} \cos \left(\frac{\sqrt{\mu}}{\Gamma(1+\alpha)}\right) \xi^{\alpha}\right) \\
\times\left(C_{1} \cos \left(\frac{\sqrt{\mu}}{\Gamma(1+\alpha)}\right) \xi^{\alpha}\right. \\
\left.\left.\left.\left.+C_{2} \sin \left(\frac{\sqrt{\mu}}{\Gamma(1+\alpha)}\right) \xi^{\alpha}\right)^{-1}\right]^{2}\right\}\right)^{1 / 2}
\end{gathered}
$$

$$
\begin{aligned}
& W_{2}(\xi)=e_{0}+\frac{2 k^{2 \alpha} f_{2}}{b_{2}} \mu \\
& \times\left[\left(-C_{1} \sin \left(\frac{\sqrt{\mu}}{\Gamma(1+\alpha)}\right) \xi^{\alpha}\right.\right. \\
& \left.+C_{2} \cos \left(\frac{\sqrt{\mu}}{\Gamma(1+\alpha)}\right) \xi^{\alpha}\right) \\
& \times\left(C_{1} \cos \left(\frac{\sqrt{\mu}}{\Gamma(1+\alpha)}\right) \xi^{\alpha}\right. \\
& \left.\left.+C_{2} \sin \left(\frac{\sqrt{\mu}}{\Gamma(1+\alpha)}\right) \xi^{\alpha}\right)^{-1}\right]^{2} \\
& +f_{2} \sqrt{\mu}\left[\left(-C_{1} \sin \left(\frac{\sqrt{\mu}}{\Gamma(1+\alpha)}\right) \xi^{\alpha}\right.\right. \\
& \left.+C_{2} \cos \left(\frac{\sqrt{\mu}}{\Gamma(1+\alpha)}\right) \xi^{\alpha}\right) \\
& \times\left(C_{1} \cos \left(\frac{\sqrt{\mu}}{\Gamma(1+\alpha)}\right) \xi^{\alpha}\right. \\
& \left.\left.+C_{2} \sin \left(\frac{\sqrt{\mu}}{\Gamma(1+\alpha)}\right) \xi^{\alpha}\right)^{-1}\right] \\
& \times\left(\sigma \left\{1+\left[\left(-C_{1} \sin \left(\frac{\sqrt{\mu}}{\Gamma(1+\alpha)}\right) \xi^{\alpha}\right.\right.\right.\right. \\
& \left.+C_{2} \cos \left(\frac{\sqrt{\mu}}{\Gamma(1+\alpha)}\right) \xi^{\alpha}\right)
\end{aligned}
$$

$$
\begin{aligned}
& \times\left(C_{1} \cos \left(\frac{\sqrt{\mu}}{\Gamma(1+\alpha)}\right) \xi^{\alpha}\right. \\
& \left.\left.\left.\left.+C_{2} \sin \left(\frac{\sqrt{\mu}}{\Gamma(1+\alpha)}\right) \xi^{\alpha}\right)^{-1}\right]^{2}\right\}\right)^{1 / 2},
\end{aligned}
$$

where $\xi=k x+c t+\xi_{0}$ and $\mu=(1 / 4) k^{-4 \alpha} \sigma b_{2}^{2}$.

In particular, if we let $C_{2}=0$ in (26)-(28), then we obtain the following solitary wave solutions, which are shown in Figures 1, 2, and 3:

$$
\begin{aligned}
& U_{3}(\xi)=\frac{1}{3} k^{-\alpha} c^{\alpha}+\frac{5}{12} k^{-2 \alpha} \sigma b_{2}^{2} \\
& -2 k^{2 \alpha} \mu\left[\tanh \left(\frac{\sqrt{-\mu} \xi^{\alpha}}{\Gamma(1+\alpha)}\right)\right]^{2} \\
& +b_{2} \sqrt{-\mu}\left[\tanh \left(\frac{\sqrt{-\mu} \xi^{\alpha}}{\Gamma(1+\alpha)}\right)\right] \\
& \times \sqrt{\sigma\left\{1-\left[\tanh \left(\frac{\sqrt{-\mu} \xi^{\alpha}}{\Gamma(1+\alpha)}\right)\right]^{2}\right\}}, \\
& V_{3}(\xi)=\frac{1}{24} \frac{b_{2}\left(16 k^{-\alpha} c^{\alpha} f_{2}+5 k^{-2 \alpha} f_{2} \sigma b_{2}^{2}-6 e_{0} b_{2}\right)}{f_{2}^{2}} \\
& -\frac{k^{2 \alpha} b_{2}}{2 f_{2}} \mu\left[\tanh \left(\frac{\sqrt{-\mu} \xi^{\alpha}}{\Gamma(1+\alpha)}\right)\right]^{2} \\
& +\frac{b_{2}^{2}}{4 f_{2}} \sqrt{-\mu}\left[\tanh \left(\frac{\sqrt{-\mu} \xi^{\alpha}}{\Gamma(1+\alpha)}\right)\right] \\
& \times \sqrt{\sigma\left\{1-\left[\tanh \left(\frac{\sqrt{-\mu} \xi^{\alpha}}{\Gamma(1+\alpha)}\right)\right]^{2}\right\}}, \\
& W_{3}(\xi)=e_{0}-\frac{2 k^{2 \alpha} f_{2}}{b_{2}} \mu\left[\tanh \left(\frac{\sqrt{-\mu} \xi^{\alpha}}{\Gamma(1+\alpha)}\right)\right]^{2} \\
& +f_{2} \sqrt{-\mu}\left[\tanh \left(\frac{\sqrt{-\mu} \xi^{\alpha}}{\Gamma(1+\alpha)}\right)\right] \\
& \times \sqrt{\sigma\left\{1-\left[\tanh \left(\frac{\sqrt{-\mu} \xi^{\alpha}}{\Gamma(1+\alpha)}\right)\right]^{2}\right\}} .
\end{aligned}
$$

If we let $C_{2}=0$ in (29)-(31), then we obtain the following periodic wave solutions, which are shown in Figures 4, 5, and 6:

$$
\begin{aligned}
U_{4}(\xi)= & \frac{1}{3} k^{-\alpha} c^{\alpha}+\frac{5}{12} k^{-2 \alpha} \sigma b_{2}^{2} \\
& +2 k^{2 \alpha} \mu\left[\tan \frac{\sqrt{\mu}}{\Gamma(1+\alpha)} \xi^{\alpha}\right]^{2}
\end{aligned}
$$




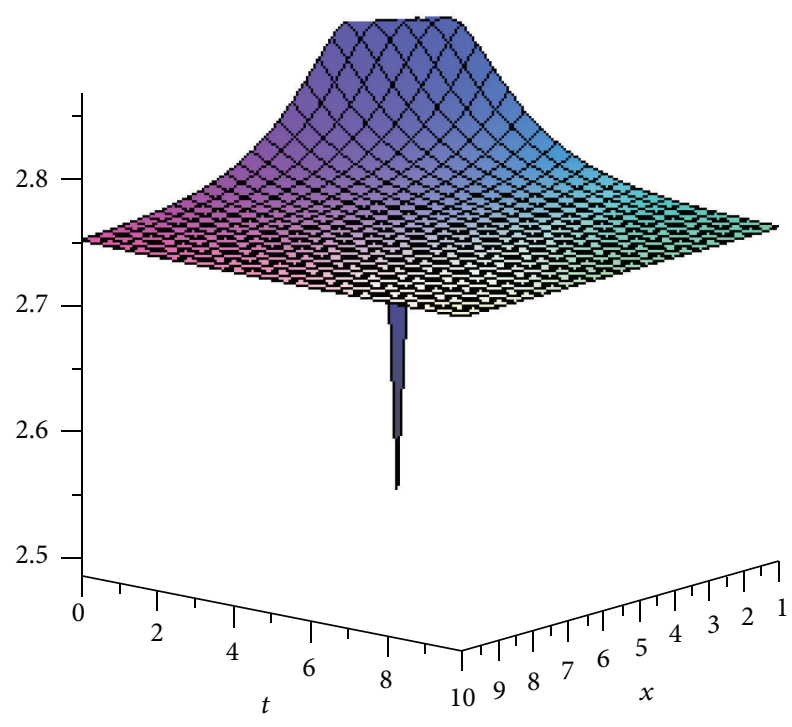

FIGURE 1: The solution $U_{3}$ in (32) with $\gamma=4 / 5, k=c=\sigma=b_{2}=1$, $\mu=-1, \xi_{0}=0$.

$$
\begin{aligned}
& -b_{2} \sqrt{\mu}\left[\tan \frac{\sqrt{\mu}}{\Gamma(1+\alpha)} \xi^{\alpha}\right] \\
& \times \sqrt{\sigma\left\{1+\left[\tan \frac{\sqrt{\mu}}{\Gamma(1+\alpha)} \xi^{\alpha}\right]^{2}\right\}}, \\
& V_{4}(\xi)=\frac{1}{24} \frac{b_{2}\left(16 k^{-\alpha} c^{\alpha} f_{2}+5 k^{-2 \alpha} f_{2} \sigma b_{2}^{2}-6 e_{0} b_{2}\right)}{f_{2}^{2}} \\
& +\frac{k^{2 \alpha} b_{2}}{2 f_{2}} \mu\left[\tan \frac{\sqrt{\mu}}{\Gamma(1+\alpha)} \xi^{\alpha}\right]^{2} \\
& -\frac{b_{2}^{2}}{4 f_{2}} \sqrt{\mu}\left[\tan \frac{\sqrt{\mu}}{\Gamma(1+\alpha)} \xi^{\alpha}\right] \\
& \times \sqrt{\sigma\left\{1+\left[\tan \frac{\sqrt{\mu}}{\Gamma(1+\alpha)} \xi^{\alpha}\right]^{2}\right\}}, \\
& W_{4}(\xi)=e_{0}+\frac{2 k^{2 \alpha} f_{2}}{b_{2}} \mu\left[\tan \frac{\sqrt{\mu}}{\Gamma(1+\alpha)} \xi^{\alpha}\right]^{2} \\
& -f_{2} \sqrt{\mu}\left[\tan \frac{\sqrt{\mu}}{\Gamma(1+\alpha)} \xi^{\alpha}\right] \\
& \times \sqrt{\sigma\left\{1+\left[\tan \frac{\sqrt{\mu}}{\Gamma(1+\alpha)} \xi^{\alpha}\right]^{2}\right\}} \text {. }
\end{aligned}
$$

Similar to the established solutions from Case 1, we can construct corresponding exact solutions to (13) from Cases $2-7$, which are omitted here.

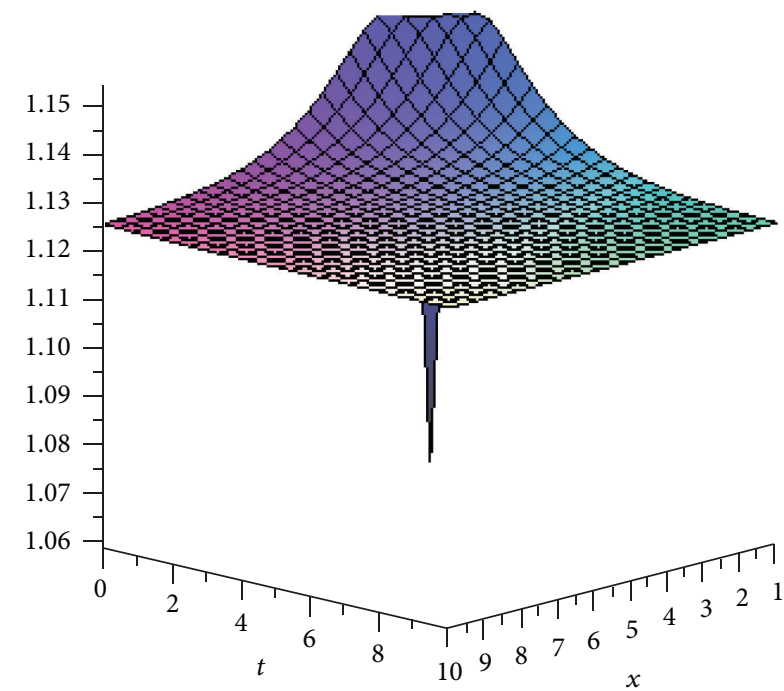

FIgURE 2: The solution $V_{3}$ in (33) with $\gamma=4 / 5, k=c=\sigma=b_{2}=$ $f_{2}=1, \mu=-1, \xi_{0}=0, e_{0}=1$.

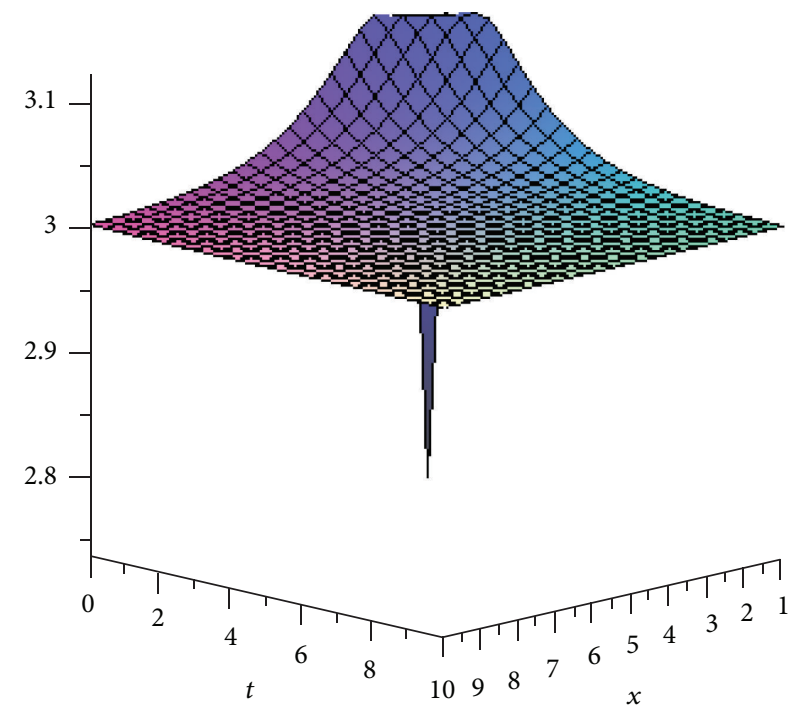

FIgURE 3: The solution $W_{3}$ in (34) with $\gamma=4 / 5, k=c=\sigma=b_{2}=$ $f_{2}=1, \mu=-1, \xi_{0}=0, e_{0}=1$.

Remark 1. We note that the solutions obtained here are of new forms compared with the solutions obtained in $[15,16]$ since a fully new method is used here.

\section{Conclusions}

Based on the concept of the modified Riemann-Liouville derivative and a variable transformation $\xi=c t+k_{1} x_{1}+$ $k_{2} x_{2}+\cdots+k_{n} x_{n}+\xi_{0}$, we have proposed a new fractional subequation method for solving fractional partial differential equations (FPDEs). By using this method, the space-time fractional generalized Hirota-Satsuma coupled KdV equations are solved successfully, and, as a result, some exact solutions are established, which may help to understand 


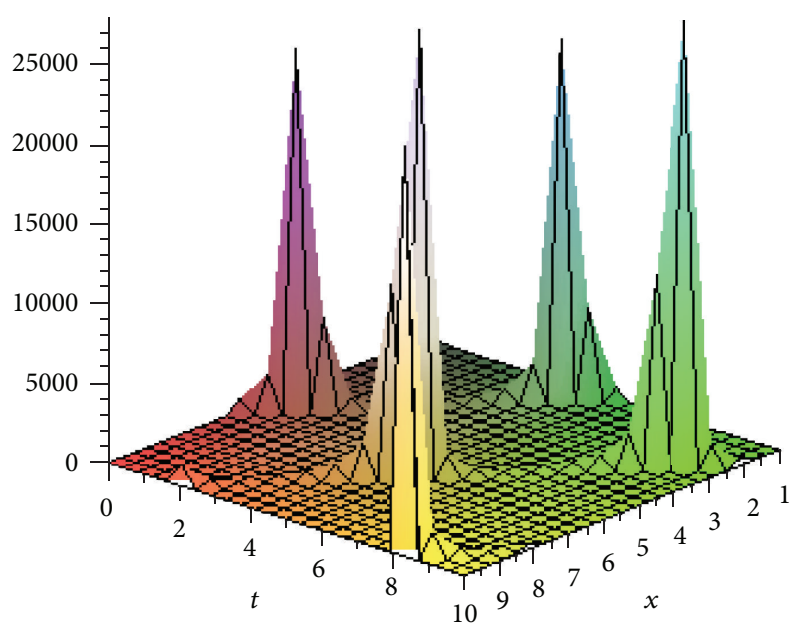

FIGURE 4: The solution $U_{4}$ in (35) with $\gamma=4 / 5, k=c=\sigma=b_{2}=1$, $\mu=1, \xi_{0}=0$.

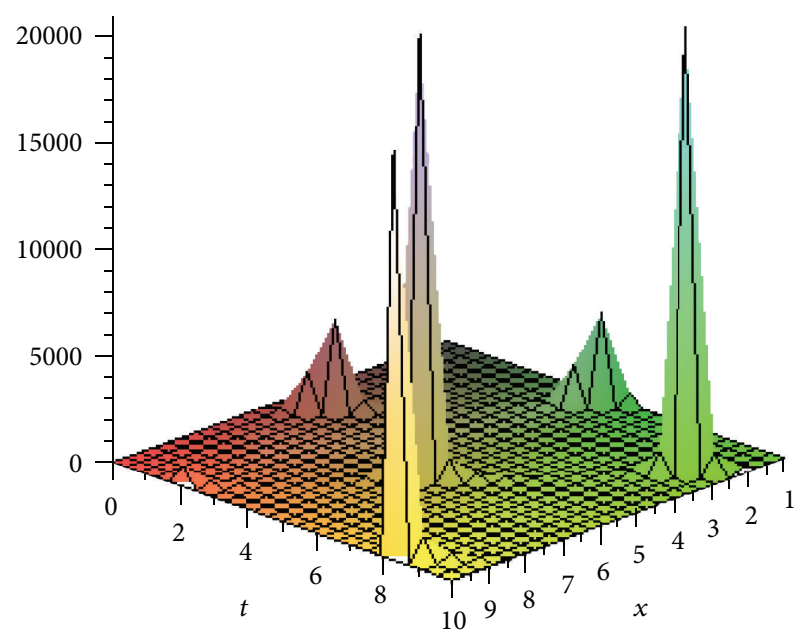

FIGURE 5: The solution $V_{4}$ in (36) with $\gamma=4 / 5, k=c=\sigma=b_{2}=$ $f_{2}=1, \mu=1, \xi_{0}=0, e_{0}=1$.

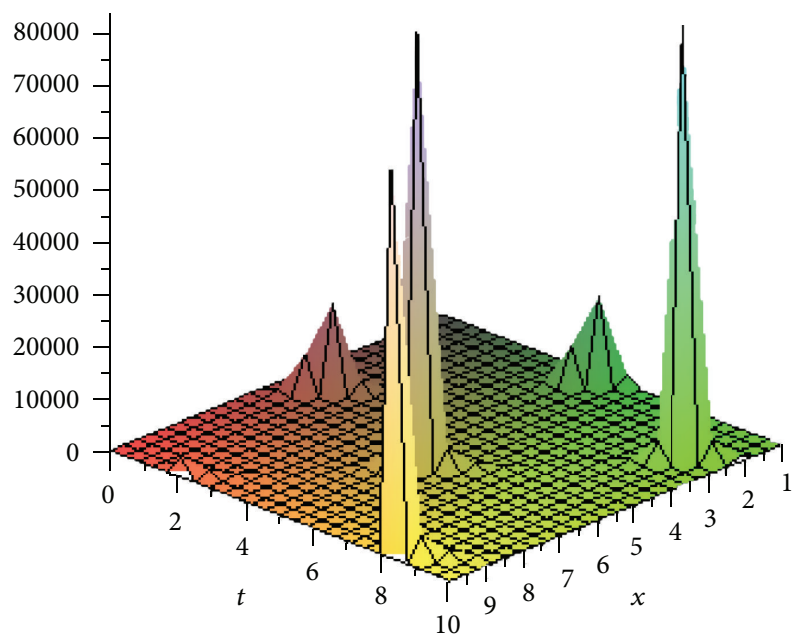

FIGURE 6: The solution $W_{4}$ in (37) with $\gamma=4 / 5, k=c=\sigma=b_{2}=$ $f_{2}=1, \mu=-1, \xi_{0}=0, e_{0}=1$. better the nonlinear wave phenomena. It is supposed that this method can be further applied to solve other FPDEs.

\section{Acknowledgments}

This work is partially supported by the National Natural Science Foundation of China (11171178) and the Program for Scientific Research Innovation Team in Colleges and Universities of Shandong Province. The authors would like to thank the reviewers very much for their valuable suggestions in the paper.

\section{References}

[1] G. C. Wu, "A fractional variational iteration method for solving fractional nonlinear differential equations," Computers \& Mathematics with Applications, vol. 61, no. 8, pp. 2186-2190, 2011.

[2] J. Ji, J. b. Zhang, and Y. J. Dong, "The fractional variational iteration method improved with the Adomian series," Applied Mathematics Letters, vol. 25, no. 12, pp. 2223-2226, 2012.

[3] G. C. Wu and E. W. M. Lee, "Fractional variational iteration method and its application," Physics Letters A, vol. 374, no. 25, pp. 2506-2509, 2010.

[4] S. Guo and L. Mei, "The fractional variational iteration method using He’s polynomials," Physics Letters A, vol. 375, no. 3, pp. 309-313, 2011.

[5] A. M. A. El-Sayed, S. H. Behiry, and W. E. Raslan, "Adomian's decomposition method for solving an intermediate fractional advection-dispersion equation," Computers \& Mathematics with Applications, vol. 59, no. 5, pp. 1759-1765, 2010.

[6] A. M. A. El-Sayed and M. Gaber, "The Adomian decomposition method for solving partial differential equations of fractal order in finite domains," Physics Letters A, vol. 359, no. 3, pp. 175-182, 2006

[7] S. S. Ray, "A new approach for the application of Adomian decomposition method for the solution of fractional space diffusion equation with insulated ends," Applied Mathematics and Computation, vol. 202, no. 2, pp. 544-549, 2008.

[8] M. Merdan, "A numeric-analytic method for time-fractional Swift-Hohenberg (S-H) equation with modified RiemannLiouville derivative," Applied Mathematical Modelling, vol. 37, no. 6 , pp. 4224-4231, 2013.

[9] M. Cui, "Compact finite difference method for the fractional diffusion equation," Journal of Computational Physics, vol. 228, no. 20, pp. 7792-7804, 2009.

[10] K. A. Gepreel, “The homotopy perturbation method applied to the nonlinear fractional Kolmogorov-Petrovskii-Piskunov equations," Applied Mathematics Letters, vol. 24, no. 8, pp. 1428$1434,2011$.

[11] Q. Huang, G. Huang, and H. Zhan, "A finite element solution for the fractional advection-dispersion equation," Advances in Water Resources, vol. 31, no. 12, pp. 1578-1589, 2008.

[12] B. Lu, "Bäcklund transformation of fractional Riccati equation and its applications to nonlinear fractional partial differential equations," Physics Letters A, vol. 376, no. 28-29, pp. 2045-2048, 2012.

[13] G. Jumarie, "Modified Riemann-Liouville derivative and fractional Taylor series of nondifferentiable functions further results," Computers \& Mathematics with Applications, vol. 51, no. 9-10, pp. 1367-1376, 2006. 
[14] S. Zhang and H. Q. Zhang, "Fractional sub-equation method and its applications to nonlinear fractional PDEs," Physics Letters A, vol. 375, no. 7, pp. 1069-1073, 2011.

[15] S. M. Guo, L. Q. Mei, Y. Li, and Y. F. Sun, “The improved fractional sub-equation method and its applications to the space-time fractional differential equations in fluid mechanics," Physics Letters A, vol. 376, no. 4, pp. 407-411, 2012.

[16] B. Zheng, " $\left(G^{\prime} / G\right)$-expansion method for solving fractional partial differential equations in the theory of mathematical physics," Communications in Theoretical Physics, vol. 58, no. 5, pp. 623-630, 2012.

[17] R. Abazari and M. Abazari, "Numerical simulation of generalized Hirota-Satsuma coupled KdV equation by RDTM and comparison with DTM," Communications in Nonlinear Science and Numerical Simulation, vol. 17, no. 2, pp. 619-629, 2012. 


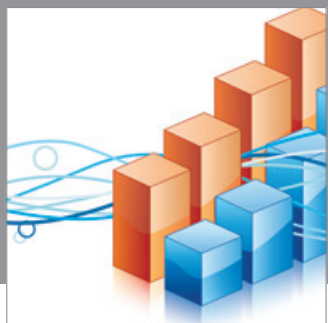

Advances in

Operations Research

mansans

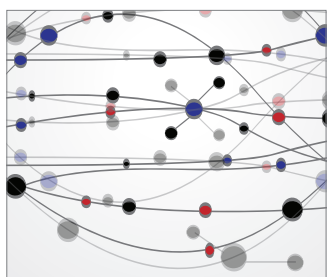

The Scientific World Journal
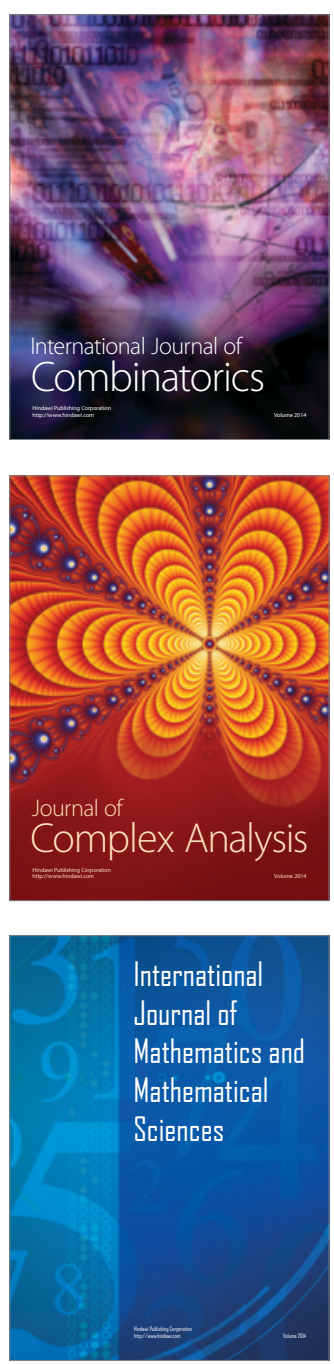
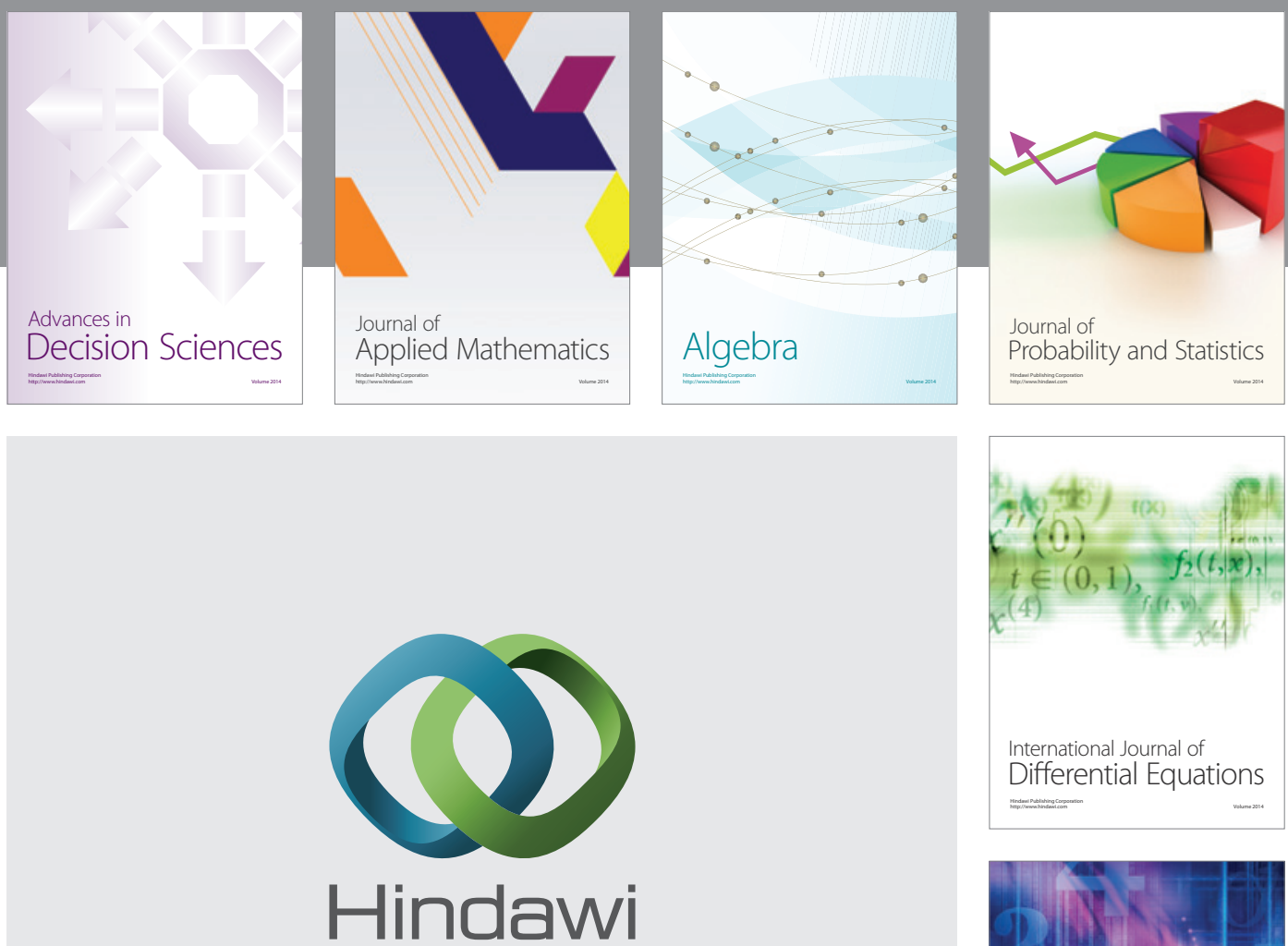

Submit your manuscripts at http://www.hindawi.com
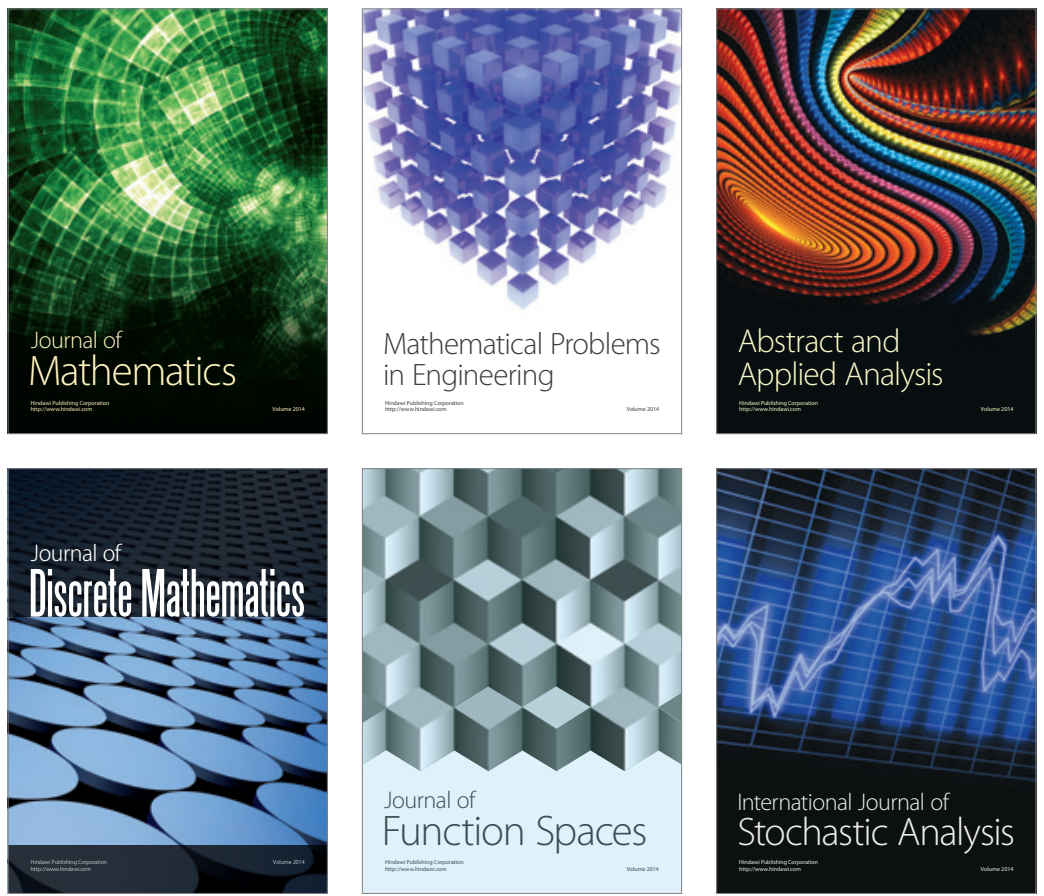

Journal of

Function Spaces

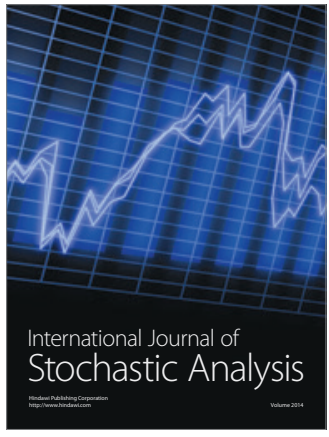

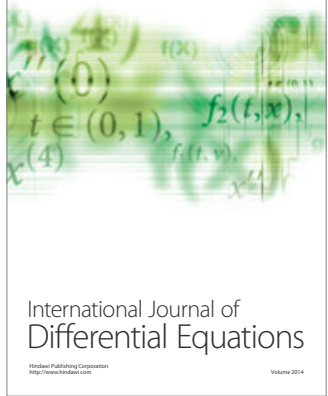
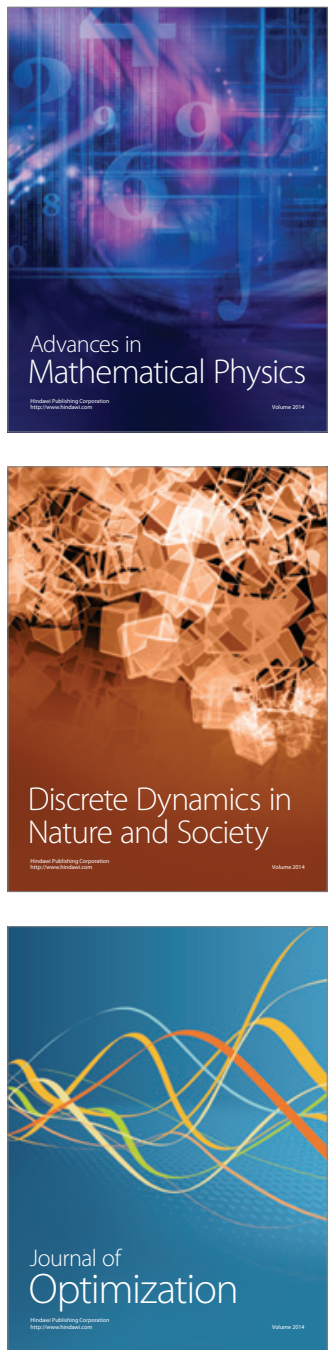